Pacific Journal of Mathematics

ORDERS WITH FINITE GLOBAL DIMENSION 


\title{
ORDERS WITH FINITE GLOBAL DIMENSION
}

\author{
MARK RAMRAS
}

Let $\Lambda$ be a left noetherian ring of finite left global dimension. Assume that $\Lambda$ is quasi-local, i.e., that $\Lambda$ modulo its Jacobson radical $J(\Lambda)$ is a simple artin ring, and suppose that for some left Ore denominator set $S$ contained in $\Lambda, \Sigma=S^{-1} \Lambda$ is a left artin ring. Then $\Sigma$ is a simple artin ring. More precisely, if $\Lambda / J(\Lambda) \cong M_{n}(K)$, the ring of $n$ by $n$ matrices over a division ring $K$, then there exists an integer $m$ dividing $n$ such that $\Lambda=M_{m}(\Delta)$ and $\Sigma=M_{m}(L)$, where $L$ is a division ring and $\Delta$ is an order in $L$.

A corollary of this is that if the above $\Lambda$ is local, then $\Sigma$ is a division ring. Another corollary is that if $F$ is a field and $\Lambda$ is a left noetherian left order in $M_{p}(F)$, and if $\Lambda$ is quasi-local and 1. gl. dim. $\Lambda<\infty$, then $\Lambda=M_{p}(R)$, where $R \subset F$ is the center of $\Lambda$.

These results were originally established by the author in a less general setting (some restrictions were placed upon the center of $\Lambda$ ). The present form of Theorem 4 and its proof are due to George Bergman, whose help is greatly appreciated.

It seems natural to ask whether it is necessary to assume that $\Lambda$ is an order in an artin ring. It is well-known that a left noetherian left hereditary ring has a left artinian left quotient ring [1]. Small [2] has given an example of a two-sided noetherian ring of global dimension two which is not an order in an artin ring. His ring is not quasi-local. We show that $\Lambda$ has an artinian left quotient ring if $\Lambda$ is quasi-local, left noetherian, and has left and right global dimension two. For rings of global dimension greater than two, the question is open.

Preliminaries, All rings under consideration have units and all modules are unital finitely generated left modules. By gl. dim. $\Lambda$ we mean the left global dimension of $\Lambda$. We now recall some standard definitions. A multiplicatively closed subset $S$, all of whose members are regular (i.e., neither left nor right zero-divisors) in $\Lambda$, is called a left Ore denominator set if the left Ore condition is satisfied: If $a \in$ $\Lambda$ and $s \in S$ then there exist $a_{1} \in \Lambda$ and $s_{1} \in S$ such that $s_{1} a=a_{1} s$. It is well-known that under this condition, $S^{-1} \Lambda=\left\{s^{-1} a \mid s \in S, a \in \Lambda\right\}$ is a ring containing $\Lambda$, and in which (clearly) the elements of $S$ are invertible. $S^{-1} \Lambda$ is called the left quotient ring (or left ring of fractions) of $\Lambda$ with respect to $S$, and $\Lambda$ is called a left order in $S^{-1} \Lambda$.

1. Some lemmas. 
Lemma 1. Let $\Gamma$ be a quasi-local artin ring of finite global dimension. Then gl. $\operatorname{dim} . \Gamma=0$, and thus $\Gamma$ is simple.

Proof. Since the global dimension of $\Gamma$ is finite, it equals the finitistic global dimension of $\Gamma$. We claim the latter is zero since $\Gamma$ is quasi-local and artin. (Though this fact is perhaps well-known, we include a proof for the sake of completeness.) It clearly suffices to show that if the projective dimension of a module $M$ is $\leqq 1$, then $M$ is projective. Since $\Gamma$ is artin, projective covers exist, and so we may assume an exact sequence $0 \rightarrow P_{1} \rightarrow P_{0} \rightarrow M \rightarrow 0$ with the $P_{i}$ 's projective and $\operatorname{Im}\left(P_{1}\right) \subset J(\Gamma) P_{0}$. Now since $\Gamma$ is artin, $J(\Gamma)$ is nilpotent, and in particular has nonzero left annihilator. So $P_{1}$ is not a faithful $R$-module. But over a quasi-local ring a nonzero projective module is a projective generator and thus faithful. Hence $P_{1}$ is zero, and $M$ is projective.

Lemma 2. Let $\Gamma$ be a left noetherian ring of finite global dimension. Let $S$ be a left Ore denominator set for $\Gamma$, and let $\Omega=$ $S^{-1} \Gamma$. Then if $P$ is a projective $\Omega$-module, there exist finitely generated projective $\Gamma$-modules $G$ and $H$ such that $P \oplus(\Omega \otimes G) \approx \Omega \otimes H$.

Proof. If $M$ is any finitely generated $\Omega$-module, there is a finitely generated $\Gamma$-module $N$ such that $M=S^{-1} N=\Omega \otimes N$. So let $P=$ $\Omega \otimes Q$, and let

$$
0 \longrightarrow F_{n} \longrightarrow \cdots F_{0} \longrightarrow Q \longrightarrow 0
$$

be a finite $\Gamma$-projective resolution of $Q$. Tensoring with $\Omega$ (which is $\Gamma$-flat) we obtain an $\Omega$-projective resolution of $P$, and since $P$ is projective, this sequence splits. Thus $P \oplus \Sigma\left(\Omega \otimes F_{2 i+1}\right) \approx \oplus \Sigma\left(\Omega \otimes F_{2 i}\right)$. Letting $G=\bigoplus \Sigma F_{2 i+1}$ and $H=\bigoplus \Sigma F_{2 i}$ yields the desired conclusion.

REMARK. In the language of Grothendieck groups, this lemma just says that the natural map $K_{0}(\Gamma) \rightarrow K_{0}(\Omega)$ is surjective. (If $R$ is a ring, $K_{0}(R)$ is the abelian group defined by one generator $[P]$ for each isomorphism class of finitely generated projective left $R$-modules $P$, and the relations $[P \oplus Q]=[P]+[Q]$.

The next lemma is a well-known consequence of Nakayama's Lemma and we omit the proof.

LEMma 3. Let $\Gamma$ be a ring. Let $\bar{\Gamma}=\Gamma / J(\Gamma)$.

(i) If $P$ and $Q$ are finitely generated projective $\Gamma$-modules such that $\bar{\Gamma} \otimes P \approx \bar{\Gamma} \otimes Q$, then $P \approx Q$.

(ii) If $G$ is a finitely generated projective $\bar{\Gamma}$-module such that 
$G \oplus(\bar{\Gamma} \otimes Q) \approx \bar{\Gamma} \otimes P$, where $P$ and $Q$ are finitely generated projective $\Gamma$-modules, then there exists a projective $\Gamma$-module $Q^{\prime}$ such that $G \approx$ $\bar{\Gamma} \otimes Q^{\prime}$ and $P \approx Q \oplus Q^{\prime}$.

2. The main results. We can now prove the main theorem of this paper.

THEOREM 4. Suppose $\Lambda$ is a quasi-local left noetherian ring with $\Lambda / J(\Lambda) \approx M_{n}(K), K$ a division ring, such that gl. dim. $\Lambda<\infty$. Suppose $S$ is a left Ore denominator set for 1 such that the left ring of fractions $\Sigma=S^{-1} \Lambda$ is artinian.

Then $\Sigma$ is simple artinian. In fact, there exists an integer $m$ dividing $n$ such that $\Lambda$ has the form $M_{m}(\Delta)$ and $\Sigma$ has the form $M_{m}(L), L$ a division ring and $\Delta$ an order in $L$.

Proof. Let $P$ denote the minimal projective module of $\Lambda / J(\Lambda)$. Then $P^{n}$ (the direct sum of $n$ copies of $P$ ) is free of rank 1 . If $Q$ is a finitely generated projective $\Lambda$-module, then $\Lambda / J(\Lambda) \otimes Q \approx P^{r}$ for some $r$. Choose $Q \neq 0$ so as to minimize $r$. Using Lemma 3, part (ii) and the fact that every finitely generated projective $\Lambda / J(\Lambda)$-module is a direct sum of copies of $P$, it is now easy to see that every finitely generated projective $\Lambda$-module is a direct sum of copies of $Q$. In particular, $\Lambda \approx Q^{m}$ for some $m$. Since $p^{n} \approx \Lambda / J(\Lambda) \approx\left(P^{r}\right)^{m}$, it follows that $n=r m$. Now let us, for the moment at least, make the convention that homomorphisms of left $R$-modules are written on the right, and composed accordingly. Then $\Lambda \cong \operatorname{End}_{\Lambda}(\Lambda) \cong \operatorname{End}_{\lrcorner}\left(Q^{m}\right) \cong$ $M_{m}(\Delta)$, where $\Delta=\operatorname{End}_{\Lambda}(Q)$. (If we write homomorphisms on the left, we should write $\Delta=\operatorname{End}_{\Lambda}(Q)^{o p p}$.)

Now consider the artin ring $\Sigma=S^{-1} \Lambda$. Note that the number of prime ideals of $\Sigma=$ the number of prime ideals of $\Sigma / J(\Sigma)=$ the rank of $K_{0}(\Sigma / J(\Sigma)$ ) (as an additive group; by the structure theory for modules over a semi-simple ring) $=$ the rank of $K_{0}(\Sigma)$ (by the lifting of idempotents). By Lemma 2 this is $\leqq$ the rank of $K_{0}(\Lambda)$, which by Lemma 3 , part (i), is $\leqq$ the rank of $K_{0}(\Lambda / J(\Lambda)$ ). But this last term is 1 since $\Lambda / J(\Lambda)$ is simple artinian. Hence $\Sigma$ has precisely one prime ideal and is thus quasi-local.

Since $\Sigma$ is left $\Lambda$-flat, gl. dim. $\Sigma \leqq$ gl. dim. $\Lambda$. By Lemma $1, \Sigma$ will now be simple artin, i.e., it will have the form $M_{m^{\prime}}(L)$ for some division ring $L$ and some integer $m^{\prime}$. We shall show that $m^{\prime}=m$. Let $V$ be the minimal projective $\Sigma$-module. Then the free $\Sigma$-module of rank 1 is isomorphic to $V^{m^{\prime}}$. We have seen above that the free $\Lambda$-module of rank 1 is isomorphic to $Q^{m}$. For some $r, \Sigma \otimes Q \approx V^{r}$. Now $V^{m^{\prime}} \approx \Sigma \cong \Sigma \otimes \Lambda \cong \Sigma \otimes Q^{m} \approx(\Sigma \otimes Q)^{m} \approx V^{r m}$. Hence $m^{\prime}=r m$. 
On the other hand, by Lemma 2, there exist integers $a$ and $b$ so that $V \oplus\left(\Sigma \otimes Q^{a}\right) \approx \Sigma \otimes Q^{b}$. Hence $V \oplus V^{r a} \approx V^{r b}$, and so $1+r a=r$ $b$. Thus $r=1$ and $m^{\prime}=m$. Therefore, we can take for the matrix units of $\Sigma$ the images of those of $\Lambda$, and this gives a map $\Delta \rightarrow L$ inducing the map $\Lambda \rightarrow \Sigma$. Since $\Lambda$ is an order in $\Sigma, \Delta$ is an order in $L$ [4, Theorem. 3.3].

Taking $n=1$ in the above theorem we get:

COROLlary 5. Suppose that $A$ is a local left noetherian ring of finite (left) global dimension and $S$ is a left Ore set for 1 such that $\Sigma=S^{-1} \Lambda$ is artinian. Then $\Sigma$ is a division ring.

Another immediate consequence of Theorem 4 is:

Corollary 6. Suppose $F$ is a field, and 1 a left noetherian order in $M_{p}(F)$. If $\Lambda$ is quasi-local and gl. dim. $\Lambda<\infty$, then $\Lambda \approx$ $M_{p}(R)$, where $R \subset F$ is the center of $\Lambda$.

Proof. Since $p$ is the matrix-rank of $M_{p}(F), p$ will be the $m$ of Theorem 4 and $F$ will be the $L$.

3. A conjecture. It would be nice to be able to remove the hypothesis, in Theorem 4 , that $\Lambda$ is an order in an artin ring. Thus we raise the

Question. Is every left noetherian quasi-local ring of finite global dimension a left order in an artin ring?

The evidence is meager. It is known that any left hereditary ring with maximum condition on left annihilators has an artinian left quotient ring (see for example [1, p. 243]). On the other hand, Small [2] has produced a two-sided noetherian ring of global dimension two which is neither a left nor a right order in an artin ring. However, this ring is not quasi-local. We conclude this paper with an affirmative answer for global dimension 2.

Proposition 7. Suppose $\Lambda$ is a left noetherian ring such that $\Lambda$ is quasi-local and left and right gl. dim. $\Lambda=2$. Then $\Lambda$ is a left order in an artin ring.

Proof. By Small [3, Theorem 2.11] it suffices to establish the regularity condition: If $x+N(\Lambda)$ is regular in $\Lambda / N(\Lambda)$ (where $N(\Lambda)$ is the maximum nilpotent ideal of $\Lambda$ ) then $x$ if regular in $\Lambda$.

So assume that $x+N(\Lambda)$ is regular in $\Lambda / N(\Lambda)$, and let $l(x)$ be the 
left annihilator of $x$ in $\Lambda$. Since the sequence

$$
0 \longrightarrow l(x) \longrightarrow \Lambda \stackrel{x}{\longrightarrow} \Lambda \longrightarrow \Lambda / \Lambda x \longrightarrow 0
$$

is exact and gl. $\operatorname{dim} . \Lambda=2, l(x)$ is $\Lambda$-projective.

Now by the regularity of $x+N(\Lambda)$ in $\Lambda / N(\Lambda)$, we have $l(x) \leqq N(\Lambda)$. So, as $N(\Lambda)$ is nilpotent, $l(x)$ has nonzero left annihilator. But, as we observed in the proof of Lemma 1, a nonzero projective module over a quasi-local ring is faithful. Hence $l(x)=0$, that is, $x$ is left regular in 1 . We have not used the one-sided Noetherian hypothesis in establishing left regularity (it is wanted only for applying Small's result), so by the same argument $x$ is right regular, as required.

\section{REFERENCES}

1. A. W. Goldie, The Structure of Noetherian Rings, Lectures on rings and modules, Springer-Verlag, 246, 213-321.

2. L. W. Small, On some questions in noetherian rings, Bull. Amer. Math. Soc., 72 (1966), 853-857.

$3 . \quad$ Orders in artinian rings, J. Algebra, 4 (1966), 13-41.

4. J. C. Robson, Artinian quotient rings, Proc. London Math. Soc., 17 (1967), 600-616. $600-616$.

Received October 18, 1972 and in revised form June 15, 1973.

BOSTON COLLEGE 



\section{PACIFIC JOURNAL OF MATHEMATICS}

\section{EDITORS}

RICHARD ARENS (Managing Editor)

University of California

Los Angeles, California 90024

\section{R. A. Beaumont \\ University of Washington \\ Seattle, Washington 98105}

\section{J. DugundjI*}

Department of Mathematics University of Southern California Los Angeles, California 90007

D. Gilbarg and J. Milgram

Stanford University

Stanford, California 94305

\section{ASSOCIATE EDITORS}
E. F. BeCKenBaCH
B. H. NeumanN
F. WOLF
K. YoSHIDA

\section{SUPPORTING INSTITUTIONS}

\author{
UNIVERSITY OF BRITISH COLUMBIA \\ CALIFORNIA INSTITUTE OF TECHNOLOGY \\ UNIVERSITY OF CALIFORNIA \\ MONTANA STATE UNIVERSITY \\ UNIVERSITY OF NEVADA \\ NEW MEXICO STATE UNIVERSITY \\ OREGON STATE UNIVERSITY \\ UNIVERSITY OF OREGON \\ OSAKA UNIVERSITY
}

\author{
UNIVERSITY OF SOUTHERN CALIFORNIA \\ STANFORD UNIVERSITY \\ UNIVERSITY OF TOKYO \\ UNIVERSITY OF UTAH \\ WASHINGTON STATE UNIVERSITY \\ UNIVERSITY OF WASHINGTON

$* * *$
$*$
AMERICAN MATHEMATICAL SOCIETY
NAVAL WEAPONS CENTER

The Supporting Institutions listed above contribute to the cost of publication of this Journal, but they are not owners or publishers and have no responsibility for its content or policies.

Mathematical papers intended for publication in the Pacific Journal of Mathematics should be in typed form or offset-reproduced, (not dittoed), double spaced with large margins. Underline Greek letters in red, German in green, and script in blue. The first paragraph or two must be capable of being used separately as a synopsis of the entire paper. Items of the bibliography should not be cited there unless absolutely necessary, in which case they must be identified by author and Journal, rather than by item number. Manuscripts, in duplicate if possible, may be sent to any one of the four editors. Please classify according to the scheme of Math. Rev. Index to Vol. 39. All other communications to the editors should be addressed to the managing editor, or Elaine Barth, University of California, Los Angeles, California, 90024.

100 reprints are provided free for each article, only if page charges have been substantially paid. Additional copies may be obtained at cost in multiples of 50 .

The Pacific Journal of Mathematics is issued monthly as of January 1966. Regular subscription rate: $\$ 60.00$ a year (6 Vols., 12 issues). Special rate: $\$ 30.00$ a year to individual members of supporting institutions.

Subscriptions, orders for back numbers, and changes of address should be sent to Pacific Journal of Mathematics, 103 Highland Boulevard, Berkeley, California, 94708.

PUBLISHED BY PACIFIC JOURNAL OF MATHEMATICS, A NON-PROFIT CORPORATION

Printed at Kokusai Bunken Insatsusha (International Academic Printing Co., Ltd.), 270, 3-chome Totsuka-cho, Shinjuku-ku, Tokyo 160, Japan

* C. R. DePrima California Institute of Technology, Pasadena, CA 91109, will replace J. Dugundji until August 1974. 


\section{Pacific Journal of Mathematics}

Vol. 50, No. $2 \quad$ October, 1974

Mustafa Agah Akcoglu, John Philip Huneke and Hermann Rost, A counter example to the Blum Hanson theorem in general spaces .............

Huzihiro Araki, Some properties of modular conjugation operator of von

Neumann algebras and a non-commutative Radon-Nikodym theorem

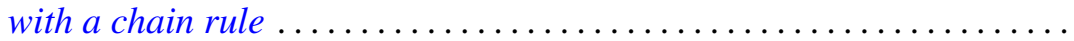

E. F. Beckenbach, Fook H. Eng and Richard Edward Tafel, Global properties of rational and logarithmico-rational minimal surfaces .....

David W. Boyd, A new class of infinite sphere packings ............. 383

K. G. Choo, Whitehead Groups of twisted free associative algebras ........

Charles Kam-Tai Chui and Milton N. Parnes, Limit sets of power series

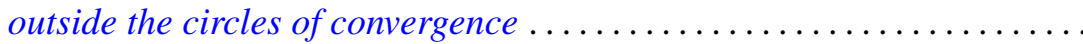

Allan Clark and John Harwood Ewing, The realization of polynomial

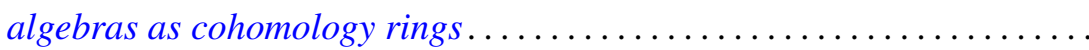

Dennis Garbanati, Classes of circulants over the p-adic and rational

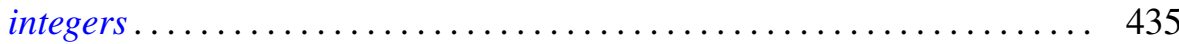

Arjun K. Gupta, On a "square" functional equation ................... 449

David James Hallenbeck and Thomas Harold MacGregor, Subordination and extreme-point theory ............................. 455

Douglas Harris, The local compactness of $v X \ldots \ldots . . . . . . . . . . . .4469$

William Emery Haver, Monotone mappings of a two-disk onto itself which fix the disk's boundary can be canonically approximated by homeomorphisms .................................. 477

Norman Peter Herzberg, On a problem of Hurwitz .................. 485

Chin-Shui Hsu, A class of Abelian groups closed under direct limits and subgroups formation ............................... 495

Bjarni Jónsson and Thomas Paul Whaley, Congruence relations and multiplicity types of algebras.....................

Lowell Duane Loveland, Vertically countable spheres and their wild sets.

Nimrod Megiddo, Kernels of compound games with simple components ....

Russell L. Merris, An identity for matrix functions ........ . .

E. O. Milton, Fourier transforms of odd and even tempered distributions ...

Dix Hayes Pettey, One-one-mappings onto locally connected generalized continua

Mark Bernard Ramras, Orders with finite global dimension

Doron Ravdin, Various types of local homogeneity. .

George Michael Reed, On metrizability of complete Moore spaces ...

Charles Small, Normal bases for quadratic extensions ..

Philip C. Tonne, Polynomials and Hausdorff matrices.... . . 